\title{
Users' Preferences for Answer Forms to Reference Questions in Libraries
}

\author{
Tomohiro Furusawa \\ Graduate School of Library, \\ Information and Media Studies, \\ University of Tsukuba \\ 1-2 Kasuga, Tsukuba-shi, 305-8550, \\ Japan \\ s1521641@u.tsukuba.ac.jp
}

\begin{abstract}
Reference service is one of the significant user support services in libraries, and in the service, the librarian provides appropriate information to a user's needs. Studies of a social question and answer (Q\&A) site revealed preferences held by users for an answer in terms of subject relevance or prediction. In order to provide a higher quality service, we investigated users' preferences for answers given in libraries. To understand what users prefer for answers, we defined four "answer forms" from the perspectives of the "amount of information" and "whether it includes any explanations of information resources or not." Respondents ranked four answers, which were developed from the answer forms in response to reference questions. The purpose was to discover their preferred way to be answered in the reference service. Results indicated that people prefer an answer, which provides multiple information resources and attached explanations of the resources, rather than an answer that only gives information resources without offering explanations. In addition, we found relationships between answer preferences and user attributes, such as age and frequency of library use.
\end{abstract}

\section{Categories and Subject Descriptors}

H.3.3 [Information Search and Retrieval]: Selection process

\section{General Terms}

Human Factors.

\section{Keywords}

Reference Service, Social Q\&A Site, User's Preference, Libraries

\section{INTRODUCTION}

Libraries have provided a reference service, in which librarians support users to solve their problems. In the reference service, "the role of the librarian is to clarify the need or complete the question" [1] being asked by users. It is most important for reference librarians to understand the various information needs of individual users and to respond to these queries appropriately. Therefore, the reference service is also defined as a form of "information consultations in which library staff recommend, interpret, evaluate, and/or use information resources to help others to meet particular information needs." [2]

This work is licensed under a Creative Commons Attribution-NonCommercial-NoDerivs International 4.0 License.

Copyright is held by the owner/author(s). ii $W A S$ '16, November 28-30, 2016, Singapore, Singapore ACM 978-1-4503-4807-2/16/11.

http://dx.doi.org/10.1145/3011141.3011168

\author{
Tetsuji Satoh \\ Faculty of Library, Information and \\ Media Science, University of Tsukuba \\ 1-2 Kasuga, Tsukuba-shi, 305-8550, \\ Japan \\ satoh@ce.slis.tsukuba.ac.jp
}

In this service, librarians should supply suitable materials that meet the information needs of users. There is, however, no one right answer or response for a user's question. Rather, "there are no specific rules, no well-beaten paths, no completely sound response. Almost everything depends on the situation, the individual, and the particular time and place." [3] In this context, it can be challenging for reference librarians to accept that there are so-called "user's preference" answers that need to be made available in the reference service.

Concurrently, the advent of the Internet has heralded the growing popularity of social question and answer (Q\&A) sites. Social Q\&A sites are "dedicated platforms for online users to ask and answer questions, browse the corpus of already answered questions, and comment and rate the quality of answers as well as vote for the best answers" [4]. There are various studies of users' preferences by analyzing questions posed and answers given on these sites. For example, [5] identified the selection criteria people employ when they choose the best answers on social Q\&A sites, and explained that the user regards some socio-emotional aspects (factors) of answers as important. Authors in [6] examined the factors that affect the selection of the best answer in a social Q\&A site, completeness, presentation, reliability, and accuracy. Thus, it has become possible to clarify factors that people expect for an answer, based on the criteria for selection of the best answer in social Q\&A sites.

In the field of library reference services, there has been a lack of focus on users' preferences on answers, seen on studies of social Q\&A sites. This is due to the effort required from librarians to provide appropriate information for "individual" users, as mentioned before. In other words, focusing on "individuality" or differences between each user is the most significant factor for reference librarians. We, however, thought that we could find out what users expect in terms of answers in reference services, as well as the studies about social Q\&A sites. Therefore, in this study, we explored users' preferences seen on answers to reference questions.

The contribution of this work is the discovery of factors that determine user preferences for an answer. This work provides the overall trends of the users' preferences to the librarians who have learned about the users from their experiences so far, therefore, it could contribute the improvement in the quality of reference services.

To reveal users' preferences for an answer, an alternative approach is required from analyses of effective log data seen in studies about social Q\&A sites, as there is no useable data about reference services available in Japan. Therefore, to clarify what kind of answers people prefer, we investigated the preferences held by people seeking answers within the service. For the 
investigation, we introduced two perspectives that were used in studies about reference services [7] [8], in order to estimate the preferences of reference service users in seeking answers. We defined four "answer forms" that reflect the preference of the user for an answer in using a reference service. In this study, as we investigated preference in terms of these answer forms, we were able to reveal factors that the user expects for an answer.

From the investigation results, we analyzed what type of answer form the user prefers, and clarified users' preferences in accessing the reference service. There are relationships between these preferences and user attributes, such as gender, age, or frequency of library use. In a previous study [9], from the investigation results of "the most preferred answer forms," we found that people prefer a "recommendation" answer and a "direction" answer to an "enumeration" answer, and high frequency library users prefer "brief" types of answers. In this paper, we reexamine how people evaluate answer forms, expanding the analysis to the investigation results of "the least preferred type of answer forms."

In terms of the type of answer form that people prefer to use, the results of this study will provide information on:

(1) Users' preferences for answers to reference questions

(2) Relationships between users' preferences (most or least preferred) and user attributes, such as age, gender, the library use frequency, or use of social Q\&A sites

Our paper is organized as follows. In Section 2, related works are discussed. In Section 3, we explain the details of the investigation design. In Section 4, the results of the investigation are presented. In Section 5, we discuss the results from the investigation. In Section 6, we conclude our research and describe implications and areas of further research.

\section{RELATED WORK}

In this study, we explore users' preferences in answers to reference questions, by using idea (a framework) of studies of social Q\&A sites. It relates to researches both reference services in libraries and users' preferences for answers of social Q\&A sites.

\subsection{Reference Services in the Libraries}

At a library, it is one of the primary tasks for librarians to provide reference services, when directly asked by users for assistance. Studies about reference services have used fieldwork, interviews, and observations among other tools as their research methodology, because these techniques are able to reveal individual characteristics of reference services. The authors in [10] analyzed conversational data between the user and the reference librarian, and presented a hypothesis about the cognitive processes used by reference librarians. The author in [11] used observation and interviewing techniques, and showed that the reference counter is characterized by a knowledge sharing perspective. [12] used participant observation, unobtrusive observation, and interviews in their research methods, highlighted the structuring and mediating role of objects in the enactment of reference work. The research design was an ethnographic case study in [11] and [12].

Virtual reference services have also become widely available in recent years. Virtual reference services allow patrons to communicate with staff without being physically present by using a computer or other Internet technology. According to [13], the terms "Virtual Reference" and "chat reference services" can be used interchangeably. Various studies use data from virtual reference services in order to analyze characteristics of the services. For example, there are studies about virtual reference services that reveal the timing of accessing the service, the original access point, who uses the service, and the types of users they are. These investigations are conducted by using access logs [14] [15] [16] [17]. Authors in [18] analyzed data from over 600 sessions and showed that the most frequently asked question was concerned with whether the library had specific items, and this observation offered valuable insights into the characteristics of users and their use of the service. Other studies have focused on not only the users' questions, but also their exchanges with librarians. Authors in [19] reviewed online reference transcripts, including virtual reference transactions and Instant Messaging transactions, and suggested that virtual reference questions tend to be more research intensive and formal in nature, while Instant Messaging questions are less focused on academic research and are informal exchanges. These studies definitely analyzed a reference service from the viewpoint of its users, however, could not reveal users' preferences for answers in reference questions, because only the answers that librarians thought to be good for users were picked up in these studies.

By contrast, the following studies utilized questionnaires in conducting their research for revealing users' awareness in reference services. [8] investigated users' preference for how the amount of materials was given in order to evaluate the service. To reveal the desires and preferences of the "Internet generation" students, the survey was conducted through the internet, and it was shown that certain research assistance options (chat, Facebook, and course management software) were not preferred options among 18-22 year-olds in the study group [20]. These studies tried to clarify users' preferences, however they seems to be still not enough to clarify the overall trend of users' preferences.

\subsection{Factors Users Expect for Answers in Social Q\&A Sites}

There are many studies that have analyzed the best answers given on social Q\&A sites, as well as the preferences of users in posing a query on these platforms.

Authors in [5] derived and grouped the best-answer selection criteria into seven value categories, and revealed that the socioemotional value of the response was particularly prominent, especially when people asked for opinions and suggestions from the service. Authors in [4] investigated the interplay between "answer quality" and "answer speed," and found that the best answers had better overall answer quality than rapidly presented responses, though they generally took longer to arrive.

The factors that the user expects from an answer, such as the prediction of the best answer on a social Q\&A site, have been revealed. [21] evaluated and predicted the quality of an answer in a social Q\&A site, and revealed that contextual information, such as a user's profile, can be critical in evaluating and predicting content quality. Authors in [6] examined the predictors of highquality responses, and found that high-quality answers include features of positive votes, and showed the significance of contentappraisal features over social and textual features. 
To sum up, there are few researches to reveal users' preferences for answers in reference services, whereas there are various studies to analysis how user choose one answer from others in social Q\&A. In this study, we revealed factors the user expects for an answer, following findings seen in studies of a social Q\&A site, in the field of reference services. Thus we directly investigated users' answer preferences by using hypothetical questions and answers as our study site.

\section{METHODOLOGY}

The survey was conducted February 8 - February 13, 2016 on the internet, in which respondents were asked to rank four "answer forms" assigned to a reference questions, according to their preference. There were 20 reference questions included in the survey. The respondents were also asked to assume that they would receive the reference service in a library, which means they would get answers from a librarian. The purpose of this study was concerned with considering preferences within reference service in libraries, rather than on general Q\&A services.

\subsection{Respondents}

Three hundred people between the ages of 15 and 79 years in Japan completed the questionnaire. These respondents were selected from 10,000 adults, and we used a commercial online survey service for this process. The questions for screening were as follows: (1) frequency of library use, (2) frequency of social Q\&A site use, (3) whether they are a librarian or not. These screening questions were asked as we considered that there is a relationship between the frequency of use of libraries, and the frequency of use of a Q\&A site. We also focused on revealing the preferences of the person presenting the question, rather than those of the person providing the response. Through the screening process, we were able to exclude people who identified themselves as librarians. Then we formed four groups of respondents by using the combination of high or low library and social Q\&A site use frequency, and randomized 75 respondents from each group. In this study, we defined high frequency of use as "to use a library or a social Q\&A site more than once a week."

\subsection{The Definition of Answer Forms}

We defined four answer forms, as seen in Table 1. These answer forms are all responses given to reference questions, and are defined from the perspectives of the "amount of information" and "whether it includes any explanations of information resources or not." There are augmentations related to these two points, which is why we draw on both perspectives. Regarding the "amount of information," for example, authors in [22] stated that users prefer short answers because the length of answers was negatively correlated with the prediction of the best answer. However, [23] concluded that "detailedness" was a key characteristic of high-quality answers. In terms of "whether it includes any explanations of information resources or not," there are also some extensions that are worth discussing. Librarians in Japanese public libraries generally perceive that they should only provide information resources to library users. That is, they have a tendency to think that they should not provide resources with an evaluation included, but rather only provide one or more materials that meet the enquirer's needs. On the other hand, Katz notes that the librarian should evaluate, interpret or clarify the offered materials, and leaves the determination of the relevance of the material up to the individual [7]. Therefore, to assess this in this study, two additional perspectives were introduced, namely "amount of information" and "whether an answer includes any explanation of the information resources or not."

The method used in composing the answer forms is as follows. First, we divided answers into two types, from the perspective of including any explanations or not when offering information resources. We defined the former as "enumeration." In terms of the latter categories ("amount of information" and "how to explain materials"), we divided these into three types; namely "briefness," which refers to reduced amounts of information, and "recommendation" as an answer that is given when multiple sources are offered and a librarian particularly recommend one as being the most suitable. We defined "direction" as an answer that is given for multiple sources with explanations being offered for some of them. In this answer form, contents of the explanation are given as a reading order of materials and a target reader.

In an "enumeration" type answer, a questioner is given multiple materials without explanations of the materials being offered by the librarian or reference service, and the individual then chooses a material from given list. In a "briefness" type answer, a questioner is given a single material, and is not made aware of other materials that could have constituted an answer. In the "recommendations" type answer, a questioner is given multiple materials, and a librarian or reference service offers a recommendation regarding the suitability of particular materials. In a "direction" type of answer, a questioner is given multiple materials with explanations attached to some of them. Therefore, a questioner is able to obtain more explanations of materials in this form than in other answer forms. In all types of answer forms, the given materials are correct and suitable for the query presented.

Table 1. Definitions of four types of answer forms

\begin{tabular}{ll}
\hline \hline Answer Forms & Definition \\
\hline Enumeration & $\begin{array}{l}\text { Appropriate materials are given, without } \\
\text { any explanations being offered for them. }\end{array}$ \\
\hline Briefness & $\begin{array}{l}\text { Only one material is given, which a } \\
\text { librarian considers as the most appropriate } \\
\text { resource. }\end{array}$ \\
\hline Recommendation & $\begin{array}{l}\text { Appropriate materials are given, with } \\
\text { recommendation offered by a librarian. }\end{array}$ \\
\hline Direction & $\begin{array}{l}\text { Suitable materials are given with } \\
\text { explanations, such as directions about the } \\
\text { order of reading or target readers. }\end{array}$ \\
\hline \hline
\end{tabular}

\subsection{Preparation of Reference Questions and Answers for the Survey}

In this study, we used the four answers that librarians seem to regularly prepare in responding to reference questions. As reference questions used in the investigation allow the study to resemble a real reference service, we drew on reference questions collected from the Collaborative Reference Database, which records actual reference questions presented in various Japanese libraries [24]. In developing questions for this investigation, we gave special attention to the cases where multiple materials were dispensed, or explanations were included in the answer. There were 20 questions developed by the authors. For example, we used queries, such as, "I want to get any books about such and such topic" for the investigation. 
Then, four answers to each question were offered, based on answer forms seen in Table 1. When developing responses, we gave particular attention to the length of the answer, so as the respondents not to think that longer answers are accurate, whereas shorter answers are wrong. The set of questions and four answers is shown in Table 2. Answer 1, namely, an "enumeration" type of answer, only provides multiple materials without any explanations of the materials being offered. In this case, a questioner should choose a material from the given list on their own. Answer 2, namely, a "briefness" type of answer, provides a single material without other materials or information being offered. In this case, a questioner is given only one material that a librarian thinks is most appropriate, without making the users aware of other materials. Answer 3, namely, a "recommendation" type of answer, refers to instances where a librarian provides multiple materials, and then recommends one of those materials. Thus, in this case, a questioner can get information about multiple materials, and a recommendation on one of them as well. Answer 4, namely a "direction" type of answer, occurs when a librarian or service provides multiple materials, and also provides some information about these books, such as the order of reading or target readers. In this case, a questioner is able to obtain more explanations of materials in this form of answers than in others.

Table 2. Example of a set question and four answers

\begin{tabular}{ll}
\hline \hline \multicolumn{2}{l}{ Question } \\
\hline \hline Q & "I want to get any books about such and such topic." \\
\hline Answers \\
\hline A.1 & $\begin{array}{l}\text { We have following books, Book A, Book B, Book C, } \\
\text { Book D and Book E - (Enumeration) }\end{array}$ \\
\hline A.2 & $\begin{array}{l}\text { Here is the Book A, which has a description of the } \\
\text { information you wanted - (Briefness) }\end{array}$ \\
\hline A.3 & $\begin{array}{l}\text { Book A is the best for you because it has easy-to- } \\
\text { understand content you wanted, among Book A, Book }\end{array}$ \\
B, Book C and Book D - (Recommendation)
\end{tabular}

\section{RESULTS}

\subsection{Overall Trends of Preferences in terms of Answer Forms for Reference Questions}

The "answer forms" ratio ranked at the top by participants in this study is shown in Table 3. The ratio of "recommendation" and "direction" are $30.7 \%$ and $30.2 \%$ respectively. Therefore, "recommendation" and "direction" are the most preferred answer forms for users. This result indicated that people prefer "to be given materials with recommendations about one of the items, or explanations of some of them," but do not prefer "to be given materials and to select the most appropriate from them," or "to be given only one material."

The "answer form" ratio that respondents ranked at the bottom is shown in Table 4. It indicates the number of answers which respondents selected as a least preferred. "Enumeration" $(42.2 \%)$ is the most frequent value, and "briefness" $(29.2 \%)$ is the secondmost frequent value in terms of being the least preferred "answer forms." These results indicated that people do not prefer "to be given materials and to select the most appropriate by themselves" or "to be given only one material." The least preferred answer forms with the smallest proportions were "recommendation" $(12.0 \%)$ and "direction" (16.6\%)

Table 3. Most Preferred

\begin{tabular}{llr}
\hline \hline Answer Forms & No. & $\%$ \\
\hline Enumeration & 1,086 & $18.1 \%$ \\
\hline Briefness & 1,263 & $21.1 \%$ \\
\hline Recommendation & 1,839 & $30.7 \%$ \\
\hline Direction & 1,812 & $30.2 \%$ \\
\hline Total & 6,000 & $100.0 \%$ \\
\hline \hline
\end{tabular}

Table 4. Least Preferred

\begin{tabular}{lrr}
\hline \hline Answer Forms & \multicolumn{1}{c}{ No. } & \multicolumn{1}{c}{$\%$} \\
\hline Enumeration & 2,533 & $42.2 \%$ \\
\hline Briefness & 1,754 & $29.2 \%$ \\
\hline Recommendation & 717 & $12.0 \%$ \\
\hline Direction & 996 & $16.6 \%$ \\
\hline Total & 6,000 & $100.0 \%$ \\
\hline \hline
\end{tabular}

People's preference for answer forms become more remarkable in the analysis of the combination of the most preferred "answer form," and the least preferred one. Figure 1 shows the bipartite graph of the combination of answer forms that people most and least preferred. Left-side nodes of the figure are answer forms that respondents ranked at the top, and right-side nodes are those at the bottom. The number in parentheses following each answer forms shows whether the "answer form" was ranked at the top or at the bottom by the respondents. Numbers on the edges that connect each node show that the number of one answer form pair was ranked at the top with another answer form ranked at the bottom. For example, in figure 1, 491 of 1,086 respondents who ranked "enumeration" at the top also ranked "briefness" at the bottom. Totally, 232 of them ranked "recommendation" at the bottom, and 363 of them ranked "direction" at the bottom.

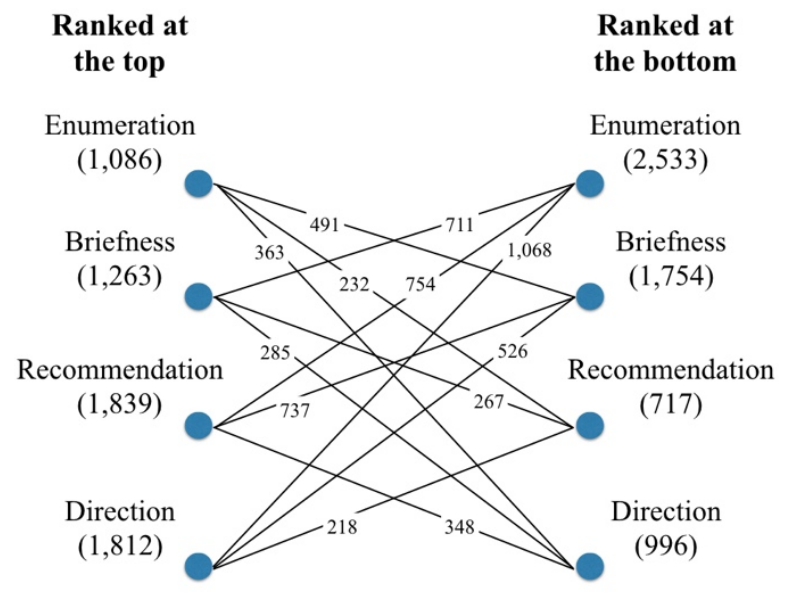

Figure 1. Combination of the most preferred answer forms and least preferred answer forms 
"Enumeration" ranked at the top $(1,086)$, while the ratio of "briefness" ranked at the bottom $(45.2 \%=491 / 1,086)$ with both respectively showing the biggest value. This indicated that about half of respondents who prefer "enumeration" not prefer "briefness." "Briefness," "recommendation," and "direction" ranked at the top $(1,263,1,839,1,812$ respectively), while the ratio of "enumeration," which is ranked at the bottom has the highest value. However, while "briefness" and "direction" ranked at the top, the ratio of "enumeration" is over half of the top ranked, though among "recommendation" being ranked at the top, the ratio of "enumeration" and "briefness" are similarly high. Therefore, people's preference for an answer in terms of the combination has characteristics of people who prefer "briefness" or "direction," rather than "enumeration," and people who prefer "recommendation" rather than "enumeration" and "briefness" in a similar manner.

\subsection{Relationships between Age or Gender and Answer Forms Preferences}

Table 5 shows the count results of the most preferred answer forms in terms of age. While people over the age of 30 show preferences for answer forms that are similar to the overall trend, ratios of all answer forms are similar in people under the age of 30 (teenagers and those in their twenties). In other words, the ratio of "enumeration" and "briefness" is higher in people under the age of 30 than in any other age group. Therefore, people under the age of 30 have characteristic tendencies that are similar to several people in that they prefer an answer in the form of "being given materials without explanations offered" or "to be given only one material."

Table 5. Preference along age group

\begin{tabular}{|c|c|c|c|c|c|c|}
\hline & & $\begin{array}{l}\text { Enum- } \\
\text { eration }\end{array}$ & $\begin{array}{c}\text { Brief- } \\
\text { ness }\end{array}$ & $\begin{array}{c}\text { Recomm- } \\
\text { endation }\end{array}$ & $\begin{array}{c}\text { Direc- } \\
\text { tion }\end{array}$ & Total \\
\hline \multirow{2}{*}{$\begin{array}{l}\text { Ages } \\
10-29\end{array}$} & No. & 92 & 95 & 77 & 96 & 360 \\
\hline & $\%$ & $25.6 \%$ & $26.4 \%$ & $21.4 \%$ & $26.7 \%$ & $100.0 \%$ \\
\hline \multirow{2}{*}{$\begin{array}{l}\text { Ages } \\
30-39\end{array}$} & No. & 166 & 261 & 422 & 431 & 1,280 \\
\hline & $\%$ & $13.0 \%$ & $20.4 \%$ & $33.0 \%$ & $33.7 \%$ & $100.0 \%$ \\
\hline \multirow{2}{*}{$\begin{array}{l}\text { Ages } \\
40-49\end{array}$} & No. & 370 & 436 & 580 & 494 & 1,880 \\
\hline & $\%$ & $19.7 \%$ & $23.2 \%$ & $30.9 \%$ & $26.3 \%$ & $100.0 \%$ \\
\hline \multirow{2}{*}{$\begin{array}{l}\text { Ages } \\
50-59\end{array}$} & No. & 257 & 262 & 475 & 466 & 1,460 \\
\hline & $\%$ & $17.6 \%$ & $17.9 \%$ & $32.5 \%$ & $31.9 \%$ & $100.0 \%$ \\
\hline \multirow{2}{*}{$\begin{array}{c}\text { Ages } \\
60 \\
\text { and } \\
\text { above }\end{array}$} & No. & 201 & 209 & 285 & 325 & 1,020 \\
\hline & $\%$ & $19.7 \%$ & $20.5 \%$ & $27.9 \%$ & $31.9 \%$ & $100.0 \%$ \\
\hline \multirow{2}{*}{ Total } & No. & 1,086 & 1,263 & 1,839 & 1,812 & 6,000 \\
\hline & $\%$ & $18.1 \%$ & $21.1 \%$ & $30.7 \%$ & $30.2 \%$ & $100.0 \%$ \\
\hline
\end{tabular}

As people under the age of 30 have a characteristic tendency that similarly prefer all answer forms, figure 2 shows the bipartite graph of the combination of answer forms, which people under 30 have most and least preferred. Among the answer forms ranked at the top, the number for "recommendation" (77) is lower than the others, and the numbers for "enumeration," "briefness" and "direction" are similar (92, 95, 96 respectively). On the other hand, among the number of answer forms ranked at the bottom, the numbers for "enumeration" (106) and "briefness" (103) are higher than "recommendation" (73) and "direction" (78). From the viewpoint of answer forms ranked at the top, a trend is seen in the fact that the numbers of all answer forms ranked at the bottom are similar. However, among "briefness," which ranked at the top (95), and the ratio of "enumeration" ranked at the bottom is nearly half $(47.4 \%=45 / 95)$, and among "recommendation," which ranked at the top (77), the ratio of "briefness" ranked at the bottom is $40.3 \%(=31 / 77)$. On the contrary, from the viewpoint of answer forms ranked at the bottom, with "enumeration" ranked at the bottom (106), the ratio of "briefness" $(42.5 \%=45 / 106)$ is highest among the others. When "Direction" ranked at the bottom (78), the ratio of "briefness" $(43.6 \%=34 / 78)$ was highest among the others. These results indicated that people who prefer types of answers that are characterized by "briefness" also prefer "enumeration" types of answers least of all.

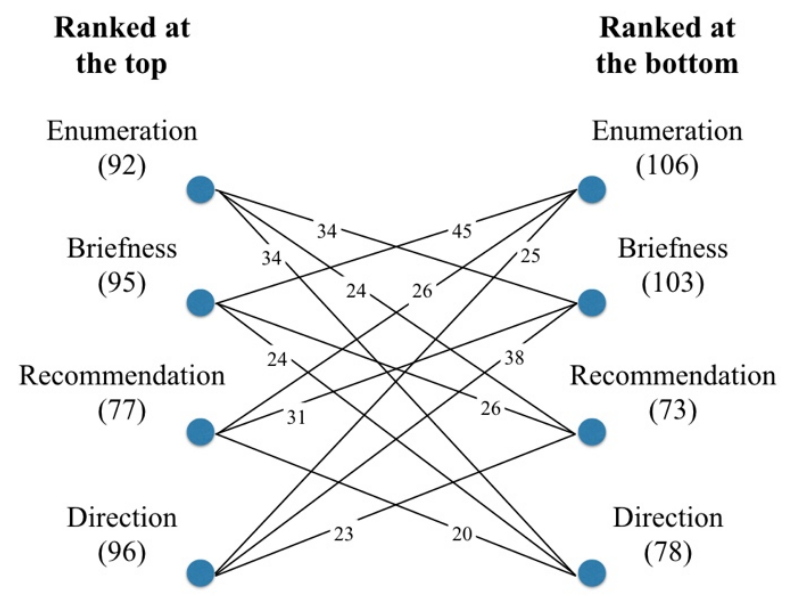

Figure 2. Combinations of the top ranked and the bottom ranked preferences in people under the age of 30 people

Here there were not significant relationships shown between gender (182 men and 118 women) and preference for answer forms in applying the chi-square test result $(\mathrm{P}=0.2328>0.01)$.

\subsection{Relationships between Use Frequency of the Library or a Social Q\&A Site and Preference for Answer Forms}

There were generally no significant relationships found between use frequency of a social Q\&A site and preference for answer forms.

Table 6 shows the two groups' preference for answer forms, separated by how frequently a library is used. Here, the high frequency group is using the library more than once a week, and the low frequency group is using the library less than once a week. 
There are significant differences seen when applying the chisquare test result $(\mathrm{P}=0.0001605<0.01)$. The high frequency of use group prefers "briefness" $(23.2 \%)$ more than the low frequency of use group does $(18.9 \%)$. From the results of the combination between answers ranked at the top and the bottom, there were similarly trends shown among people who prefer "briefness" rather than "enumeration."

Table 7 shows the ratio that the two groups least preferred in terms of preference of answer forms, separated by use frequency of a library. There are also significant differences seen in applying the chi-square test result $\left(\mathrm{P}=2.186 \times 10^{-6}<0.01\right)$. From the results shown in Table 7 , it can be seen that the high frequency of use group prefers "recommendation" (12.7\%) and "direction" $(18.8 \%)$ less than the low frequency of use groups does $(11.2 \%$ and $14.4 \%$ respectively). The low frequency of use group has a higher rate for rejecting "enumeration" (44.6\%) than the high frequency of use groups $(39.8 \%)$. This result indicated that people who use libraries frequently do not prefer an answer that includes explanations, such as "recommendation" or "direction," but people who use libraries infrequently do not prefer answers that are offered without explanations attached, such as "enumeration."

Table 6. Relationships between use frequency of libraries and preference for an answer (ranked at the top)

\begin{tabular}{lrrrr}
\hline \hline \multirow{2}{*}{ Use frequency } & \multicolumn{2}{c}{ High } & \multicolumn{2}{c}{ Low / No use } \\
\cline { 2 - 5 } & No. & $\%$ & No. & \multicolumn{1}{c}{$\%$} \\
\hline Enumeration & 555 & $18.5 \%$ & 531 & $17.7 \%$ \\
\hline Briefness & 696 & $23.2 \%$ & 567 & $18.9 \%$ \\
\hline Recommendation & 881 & $29.4 \%$ & 958 & $31.9 \%$ \\
\hline Direction & 868 & $28.9 \%$ & 944 & $31.5 \%$ \\
\hline Total & 3,000 & $100.0 \%$ & 3,000 & $100.0 \%$ \\
\hline \hline
\end{tabular}

Table 7. Relationships between use frequency of the library and preference for an answer (ranked at the bottom)

\begin{tabular}{lrrrr}
\hline \hline \multirow{2}{*}{ Use frequency } & \multicolumn{2}{c}{ High } & \multicolumn{2}{c}{ Low / No use } \\
\cline { 2 - 5 } & No. & $\%$ & \multicolumn{1}{c}{ No. } & \multicolumn{1}{c}{$\%$} \\
\hline Enumeration & 1,195 & $39.8 \%$ & 1,338 & $44.6 \%$ \\
\hline Briefness & 860 & $28.7 \%$ & 894 & $29.8 \%$ \\
\hline Recommendation & 381 & $12.7 \%$ & 336 & $11.2 \%$ \\
\hline Direction & 564 & $18.8 \%$ & 432 & $14.4 \%$ \\
\hline Total & 3,000 & $100.0 \%$ & 3,000 & $100.0 \%$ \\
\hline \hline
\end{tabular}

\section{DISCUSSION}

In this study, we investigated people's preferences for an answer to a reference question, from the viewpoint of their most or least preferred answer forms.

In the overall trends, people prefer "recommendation" and "direction," but not "enumeration" and "briefness." This means that people prefer an answer to be given when receiving multiple materials with explanations offered by the librarian or reference service as a response to reference questions. Regardless of the amount of materials, people prefer an answer that includes explanations of materials, and to not simply be given the reference materials. In addition, people not only expect an answer for given materials, but they also generally rated those answer forms that exclude explanations of materials lower than others.
From the results of preferences separated by users' age, people over 30 conform to dominant trends. In contrast, people under 30 , namely teenagers and people in their twenties, prefer a greater variety of answer forms than people in other age groups. As those under 30 have a greater preference for "enumeration" as compared to those in other age groups, there is a tendency shown that people who are under 30 think that explanations of materials are not necessary. In comparison to other age groups, those under 30 have a variety of preferences for an answer. Having said this, the results of the combination of preferences for answer forms show that there is clearly a tendency for people to prefer "briefness" and not prefer "enumeration."

There are relationships shown between frequency of library use and preference for answer forms. The high frequency of use group prefers "briefness" forms of answers and that they be given only one correct material. They also show a slight disinclination for an answer that includes explanations of materials, as compared with users who access libraries less frequently. As people using the library at high frequency rates have greater opportunities to access various materials, they also not might need as much guidance to related materials from the librarian in a reference service.

\section{CONCLUSION}

To clarify factors that affect people's preferences of an answer given in response to a reference question, we investigated preferences for answer forms in a reference service. We defined four answer forms from two viewpoints in terms of "amount of information," and "whether it includes any explanations of information resources."

From the investigation results, we found valuable insights that people expect an answer with explanations of information resources, regardless of the amount of information received. An interesting result showed that teenagers and those in their twenties preferred an answer of being "given multiple materials without explanations offered for them." In addition, we revealed that people who use a library at higher frequency prefer an answer that simply provides their requested materials.

In the findings of this study, one of the most interesting point is that those in their twenties and teenagers prefer "enumeration" answers more, which means not a few people in their twenties prefer to be only given materials without explanations, and their preferences are more various than other ages. This age group is known as the "Google generation," as mentioned that "most visitors to scholarly sites view only a few pages, many of which do not even contain real content, and in any case do not stop long enough to do any real reading [25]," it is thought that there is the tendency they expect simplicity for an answer. On the contrary, our findings shows the diversity of their preference for an answer. Therefore, future studies should aim to further clarify reference service users' preferences, especially that of twenties. In doing so, it is possible to determine the expectations or demands people hold for libraries and these services.

\section{ACKNOWLEDGMENTS}

This work was supported by Grant-in-Aids for Scientific Research No.25280110 and 16H02904. 


\section{REFERENCES}

[1] Chu, F. 1996. Framing reference encounters, $R Q$, 36(1), 93101.

[2] American Library Association. 2008. "Definitions of Reference". January 14, 2008.

http://www.ala.org/rusa/resources/guidelines/definitionsrefer ence. (accessed 2016-07-19)

[3] Katz, W. 2002. Reference services and reference processes (8th ed.) McGraw-Hill. (125-127)

[4] Chua Alton, Y.K. and Banerjee S. 2013. So fast so good: An analysis of answer quality and answer speed in community Question - answering sites. Journal of the American Society for Information Science and Technology, 64(10), 2058-2068.

[5] Kim, S. and Oh, S., 2009. Users' relevance criteria for evaluating answers in a social Q\&A site. Journal of the American Society for Information Science and Technology, 60(4), 716-727.

[6] Blooma, M.J., Hoe-Lian Goh, D. and Yeow-Kuan Chua, A., 2012. Predictors of high-quality answers. Online Information Review, 36(3), 383-400.

[7] Katz, W. 2002. Reference services and reference processes (8th ed.) McGraw-Hill. (156-157)

[8] Crawford, G.A., 1994. A conjoint analysis of reference services in academic libraries. College and Research Libraries, 55, 257-257.

[9] Furusawa, T., Matsubayashi, M., Satoh, T. 2016, June. Expectation of the General People for Answer Forms to a Reference Question. Proceedings of 2016 Japan Society of Library and Information Science Spring Conference. 51-54.

[10] Ikeya, N., 1990. The Reference Librarians' Use of Knowledge and Decision Making: The Analysis of User/Librarian Interaction. Library and information science, 28, 81-103.

[11] Cavanagh, M.F., 2006. Re-conceptualizing the 'Reference Transaction'- the Case for Interaction and Information Relationships at the Public Library Reference Desk. Canadian Journal of Information and Library Sciences, 30(1/2), 1-19.

[12] Cavanagh, M.F., 2013. Interpreting reference work with contemporary practice theory. Journal of Documentation, 69(2), 214-242.

[13] Granfield, D. and Robertson, M., 2008. Preference for reference: new options and choices for academic library users. Reference \& User Services Quarterly, 44-53.
[14] Sears, J., 2001. Chat reference service: An analysis of one semester's data. Issues in science and technology librarianship, 32, 200-6.

[15] Radford, M.L., 2006. Encountering virtual users: A qualitative investigation of interpersonal communication in chat reference. Journal of the American Society for Information Science and Technology, 57(8), 1046-1059.

[16] Marsteller, M. and Neuhaus, P., 2001, June. The chat reference experience at Carnegie Mellon University. In Presentation at the American Library Association Annual Conference.

[17] Broughton, K.M., 2003. Usage and user analysis of a realtime digital reference service. The Reference Librarian, 38(79-80), 183-200.

[18] Kibbee, J., Ward, D. and Ma, W., 2002. Virtual service, real data: results of a pilot study. Reference services review, 30(1), 25-36.

[19] Rourke, L. and Lupien, P., 2010. Learning from chatting: How our virtual reference questions are giving us answers. Evidence Based Library and Information Practice, 5(2), 63-74.

[20] Ismail, L., 2010. What net generation students really want: Determining library help-seeking preferences of undergraduates. Reference Services Review, 38(1), 10-27.

[21] Shah, C. and Pomerantz, J., 2010, July. Evaluating and predicting answer quality in community QA. In Proceedings of the 33rd international ACM SIGIR conference on Research and development in information retrieval (411418). ACM.

[22] Blooma, M. J., Chua, A. Y. K., Goh, D. H. L. 2010. Selection of the best answer in CQA services. In Information Technology: New Generations (ITNG), April, 2010 Seventh International Conference (534-539). IEEE.

[23] Ishikawa, D., Sakai, T., Seki, Y., Kuriyama, K., Kando, N. 2011. Automatic Prediction of High-Quality Answers in Community QA. Journal of Japan Society of Information and Knowledge, 21(3), 362-382

[24] National Diet Library. 2005. Collaborative Reference Database. http://crd.ndl.go.jp/reference/. Retrieved July 31, 2016

[25] Rowlands I., Nicholas D., Williams P., Huntington P., Fieldhouse M., Gunter B., Tenopir C. 2008, July. The Google generation: the information behaviour of the researcher of the future. Aslib proceedings, 60(4) 290-310 